\title{
MULTIDIMENSIONAL BIOACTIVITY PROFILING IN NATURAL PRODUCT - BASED DRUG DISCOVERY
}

\section{(C) Tammela $P$.}

\author{
Centre for Drug Research, Faculty of Pharmacy, University of Helsinki, P. O. Box 56, FI-00014 \\ University of Helsinki, Finland
}

The discovery of novel leads with therapeutic potential is challenged by the need of chemically diverse compound libraries yielding leads with distinct biological functions, but with compatible ADMET properties. Complementing synthetic chemical libraries with natural products (NP) and using NPs as scaffolds to produce novel structures are important resources to expand chemical diversity of compound libraries for biological screening. Utilization of NP-based libraries in bioactivity screening, however, entails additional aspects that need to be considered. New advancements in screening technologies, such as coupling of microfractionation to bioactivity profiling, are making a significant impact on NP discovery timelines (1). In addition to using improved technologies in processing NPs, our recent work dem- onstrates the importance of using multidimensional bioactivity profiling and high-quality bioassays in NP-based drug discovery. Examples will be presented to describe the approach we have taken to study NP-based libraries in the context of antimicrobial drug discovery. Information obtained from simultaneous screens utilizing microbes, mammalian cells and isolated targets have provided us tools for in-depth characterization of NPs. In conclusion, evaluating the biological activity of NPs in several models in parallel provides multidimensional bioactivity profiles which are highly beneficial when considering their true potential for therapeutic applications.

Reference: (1) Vuorela, P., Leinonen, M., Saikku, P., Tammela, P., Rauha, J. P., Wennberg, T., Vuorela, H. (2004), Curr. Med. Chem. 11: 1375-1389.

\section{NOVEL BIOACTIVE POLYPHENOLS FROM DIPTEROCARPACEAE}

\section{(C) Tan Ren Xiang}

Institute of Functional Biomolecules, State Key Laboratory of Pharmaceutical Biotechnology, Nanjing University, Nanjing 210093, P. R. China. E-mail: rxtan@nju.edu.cn

Resveratrol (trans-3,5,4'-trihydroxystilbene) is unique since it exists as a phytoalexin in grapes and other plant-derived foodstuffs, and has been shown to have an array of functions (1). Our phytochemical attention to species belonging to the family Diptero-

carpaceae has resulted in the characterization of a group of unusual resveratrol aneuploids and oligomers. The structures were elucidated by comprehensive spectroscopic analyses and computational quantum chemical method.

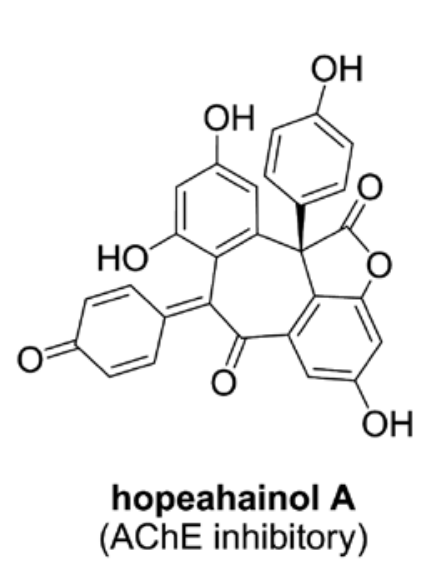

In conjunction with the bioassay, some of the novel polyphenols found in species of the family have been demonstrated to be acetylcholinesterase inhibitory (e.g., hopeahainol A), anti-inflammatory (e.g., vatiparol) and immunosuppressive (e.g., diptoindonesin G). The distinct structure and activity profile accommodate

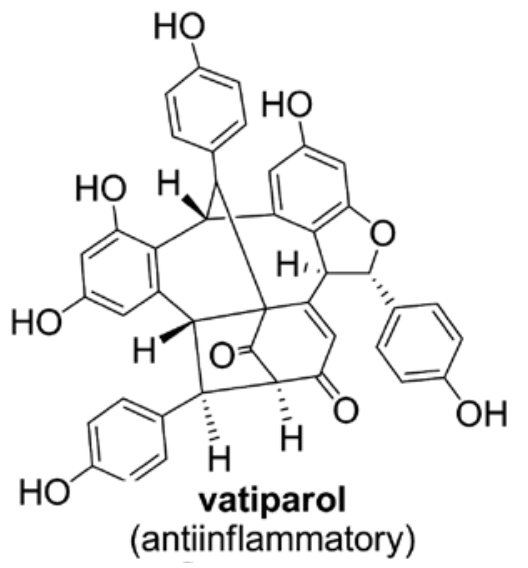<smiles>O=C1c2c(O)cc(O)cc2-c2c(-c3ccc(O)cc3)oc3cc(O)cc1c23</smiles>

diptoindonesin G (immunosuppressive)

collectively that possible starting molecules may be explored therefrom for the relevant drug discovery.

Reference: (1) H.M. Ge, H.Sun, N. Jiang, Y. H. Qin, H. Dou, T. Yan, Y. Y. Hou, C. Griesinger, R.X. Tan. Chem. Eur. J. 2012, 18, 5213-5221, and related references therein. 